\title{
Broadband Multilevel Fast Multipole Algorithm For Large-Scale Problems With Nonuniform Discretizations
}

\author{
Özgür Ergül ${ }^{*}$, Barışcan Karaosmanoğlu*, Manouchehr Takrimi ${ }^{\dagger}$, and Vakur B. Ertürk ${ }^{\dagger}$ \\ ${ }^{*}$ Department of Electrical and Electronics Engineering \\ Middle East Technical University, Ankara, TR-06800, Turkey \\ e-mails: ozgur.ergul@eee.metu.edu.tr, bariscan@metu.edu.tr \\ ${ }^{\dagger}$ Department of Electrical and Electronics Engineering \\ Bilkent University, Ankara, TR-06800, Turkey \\ e-mails: mtakrimi@bilkent.edu.tr, vakur@ee.bilkent.edu.tr
}

\begin{abstract}
We present a broadband implementation of the multilevel fast multipole algorithm (MLFMA) for fast and accurate solutions of multiscale problems involving highly nonuniform discretizations. Incomplete tree structures, which are based on population-based clustering with flexible leaf-level boxes at different levels, are used to handle extremely varying triangulation sizes on the same structures. Superior efficiency and accuracy of the developed implementation, in comparison to the standard and broadband MLFMA solvers employing conventional tree structures, are demonstrated on practical problems.
\end{abstract}

\section{INTRODUCTION}

Broadband electromagnetic solvers are required for efficient and accurate numerical solutions of multiscale problems that involve different feature sizes with respect to wavelength. Conventional formulations and solution algorithms designed for ordinary electrodynamic problems need to be converted into broadband implementations that are capable of handing complex models including both dense and coarse discretizations. A truly broadband solver must involve not only stable formulations and factorization/diagonalization routines, but also efficient methods to tackle with arbitrary mesh sizes in a single structure. In this study, we present a broadband multilevel fast multipole algorithm (MLFMA) that is based on the concept of incomplete tree structures, which are essentially required to solve challenging problems with highly nonuniform discretizations.

MLFMA [1],[2] and its diverse low-frequency implementations that employ alternative factorizations and diagonalizations are well known in the literature [3]-[14]. In addition, various broadband MLFMA implementations, which effectively combine low-frequency and high-frequency techniques for efficient and stable computations of interactions in different electromagnetic regimes, have been developed [15]-[25]. On the other hand, algorithmic routines, such as efficiently organizing near-field and far-field interactions, especially on nonuniform discretizations that arise in multiscale structures, have not been considered in sufficient depth [26]. In fact, without a multiscale construction of tree structures, broadband solvers may not provide accurate and/or efficient solutions that benefit from the true power of MLFMA.

In the next section, we briefly present and discuss the concept of incomplete tree structures for a truly broadband MLFMA solver. Section III presents numerical examples, followed by concluding remarks in Section IV.

\section{InCOMPlete TREe StRUCTURES}

We consider fast and accurate solutions of electromagnetic problems involving highly nonuniform discretizations of large structures with small but important details and features. For stable computations of interactions at all distances, the scaled diagonalization of the Green's function is employed [27]. The considered problems involve closed conductors that can be formulated with the magnetic-field integral equation (MFIE), which is stable at both low and high frequencies. Despite stable formulations (via MFIE) and stable computations of interactions (via scaled diagonalization), nonuniform discretizations bring additional challenges when standard tree structures are used. Consider such a problem discretized with $N$ unknowns, where unknowns are not distributed uniformly on the surface of the structure. The conventional approach, which relies on the collective division of computational boxes into subboxes, leads to two options.

- The number of levels can be limited, allowing for accurate computations of interactions, while this may lead to very high computational load, and even higher than linearithmic (e.g., $\mathrm{O}\left(N^{2}\right)$ ) complexity, due to overcrowded boxes containing dense discretizations.

- The number of levels can be relaxed to reach $\mathcal{O}(1)$ elements per a lowest-level box, while this may lead to inaccurate computations due to discretization elements sticking out of boxes.

There are implementations, where the second issue is addressed and the overflowed discretization elements are avoided, e.g., by clustering integration points rather than discretization elements. However, many nonuniform discretizations in practical problems that are considered in this study 
involve much difficult scenarios, in which a discretization element (triangle) can significantly be larger than the box size, even leading to far-field boxes on the same element. For such problems, inflating the near-field matrices by picking such faulty interactions is not an efficient option.

In this work, we consider incomplete tree structures, which are based on recursive clustering based on box populations. Such a strategy leads to leaf-boxes at different levels, leading to perfect packing of discretization elements with diverse sizes. Without changing the recursive structure of MLFMA, we redefine near-field and far-field rules, as detailed in [28]. The developed broadband implementations based on the incomplete tree structures have $\mathcal{O}(N \log N)$ complexity, being independent of the level of the nonuniformity in the discretization size. In addition, the incomplete tree structures automatically reduce into ordinary ones when the mesh size becomes uniform.

\section{NumericAl EXAMPLES}

As numerical examples, we consider the analysis of a water mine structure depicted in Fig. 1. The size of the structure is $0.7 \mathrm{~m}$, corresponding to nearly $\lambda / 4$ at $100 \mathrm{MHz}$. The scattering problem is discretized with the Rao-Wilton-Glisson functions on triangular domains, where the multiscale factor that is defined as the ratio of the sizes of the largest (around $\lambda / 3$ ) and smallest (around $\lambda / 1500$ ) triangles is approximately 550. As also depicted in Fig. 1, this large factor arises due to very dense discretizations used on the probes. The problem is formulated with MFIE and discretized with a total of 103,527 unknowns. Fig. 2 presents the solution of the scattering problem using three different implementations, namely, normal (conventional MLFMA), scaled (stabilized MLFMA via scaled diagonalization [27] without incomplete tree structures), and IL (proposed broadband MLFMA with incomplete tree structures). For all implementations, different numbers of levels are used, depending on the available computational hardware limits. The root-mean-square (RMS) error is calculated by comparing the far-zone electric field obtained by using the corresponding implementation with reference values obtained by using three-level (single-level translation) MLFMA. The following observations can be made.

- Using the conventional MLFMA, the error becomes $2.04 \%$ immediately when the number of levels is increased to four. A drastically jump to $14.0 \%$ occurs when the number of levels becomes six, as a combined effects of the low-frequency breakdown of the conventional diagonalization and overflowing elements out of boxes. Using five levels, leading to a reasonable $2.02 \%$ error, the processing time of the conventional MLFMA is more than 22,206 seconds.

- Using the scaled MLFMA, the error values are reduced in comparison to the conventional MLFMA, while it still increases to $6.51 \%$ for six levels due to unavoidable overflowing elements. For five levels, leading to $0.56 \%$ error, the processing time is more than 25,101 seconds due to extra costs of the scaled diagonalization [27].
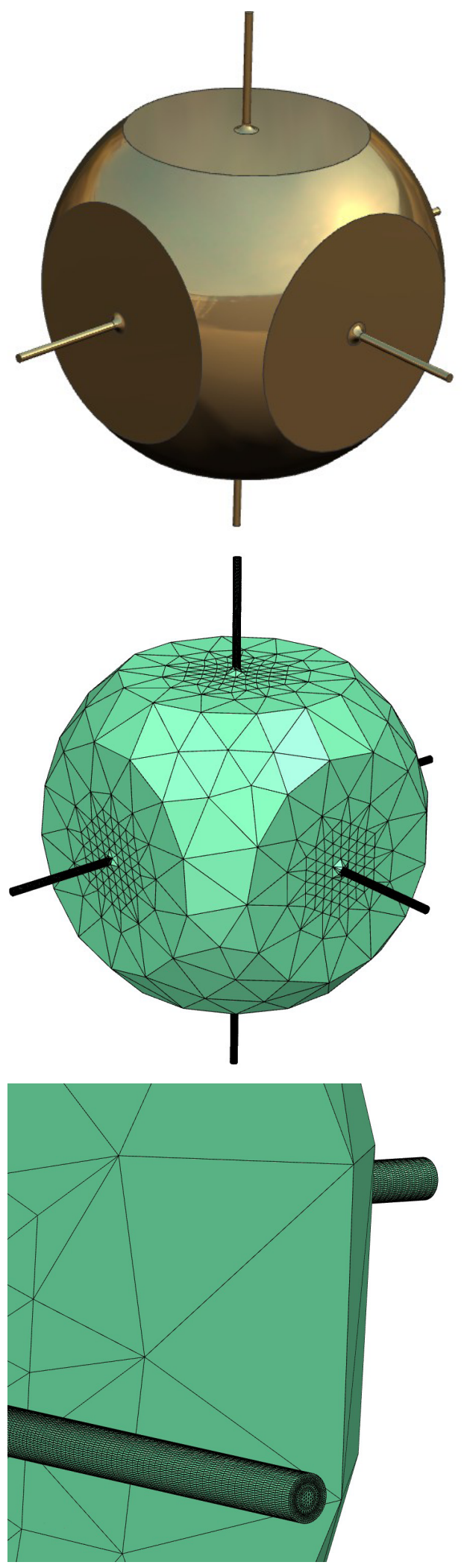

Fig. 1. A water mine structure that is discretized with triangles using a highly nonuniform mesh size. A zoomed picture is included to show the very dense discretizations of the probes. 
Water Mine, Tri: 69105, RWG: 103527, f=100 MHz

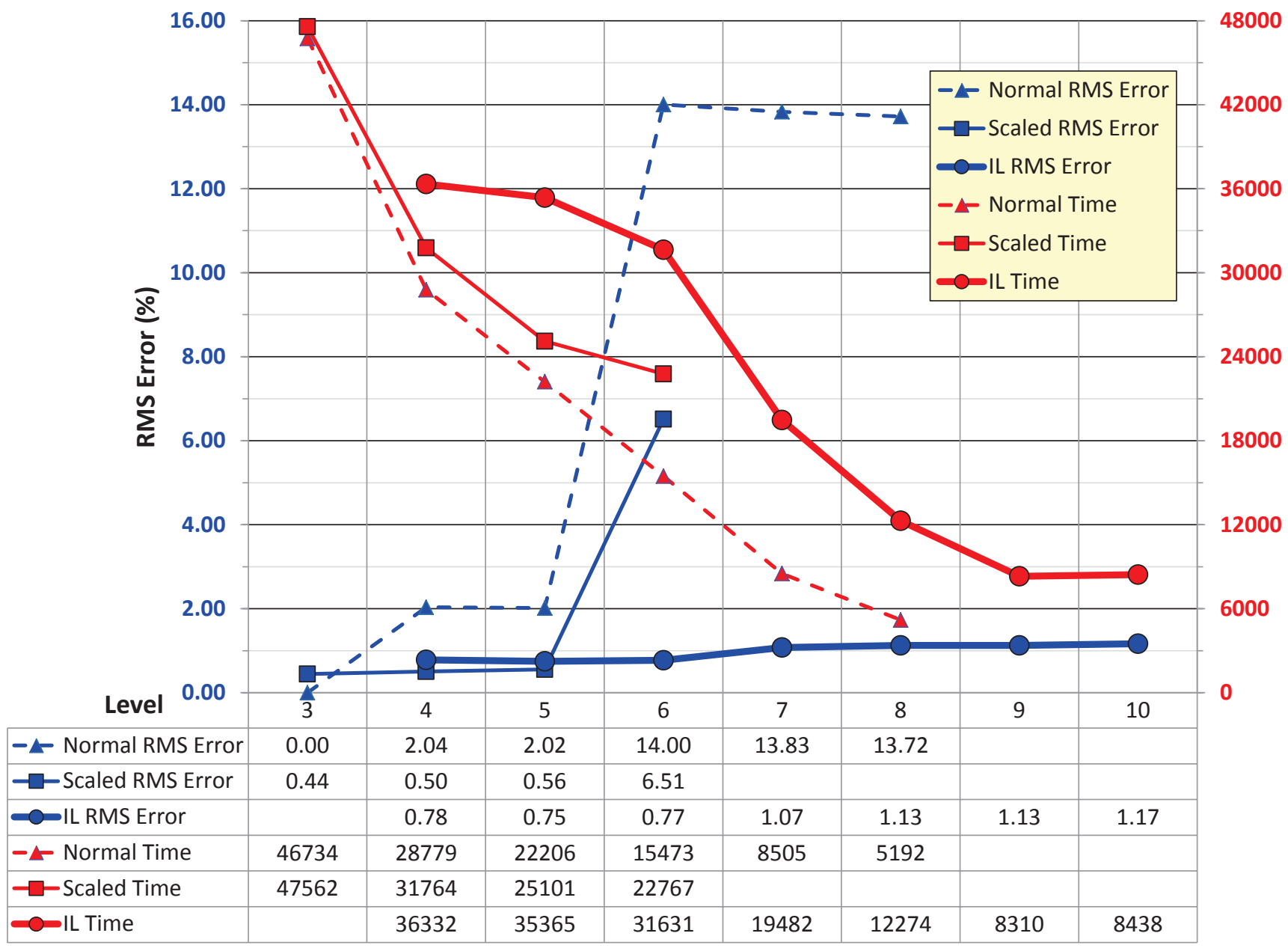

Fig. 2. Alternative solutions of the scattering problem involving the mine structure in Fig. 1.

- Using incomplete tree structures, the error is stabilized at around $1 \%$, independent of the number of levels. Specifically, the number of levels can be increased up to 10 , without any overflowing elements. This way, the efficiency of the implementation can be improved significantly, and the processing time can be reduced to less than 8500 seconds without any accuracy issues.

\section{CONCLUDING REMARKS}

A truly broadband implementation of MLFMA requires not only stable formulations and diagonalization techniques, but also broadband constructions of tree structures for efficiently partitioning nonuniform discretization elements into suitable boxes. We present an implementation based on incomplete tree structures, which allow for leaf-level boxes at different levels. By eliminating drastically large errors due to overflowing discretization elements in the conventional tree structures, the developed implementation provides both fast and accurate so- lutions of challenging problems involving highly nonuniform discretizations.

\section{ACKNOWLEDGMENT}

For this work, Özgür Ergül was supported by the Turkish Academy of Sciences (TUBA).

\section{REFERENCES}

[1] W. C. Chew, J.-M. Jin, E. Michielssen, and J. Song, Fast and Efficient Algorithms in Computational Electromagnetics. Boston: Artech House, 2001.

[2] Ö. Ergül and L. Gürel, The Multilevel Fast Multipole Algorithm (MLFMA) for Solving Large-Scale Computational Electromagnetics Problems. Wiley-IEEE, 2014.

[3] L. Greengard, J. Huang, V. Rokhlin, and S. Wadzura, "Accelerating fast multipole methods for the Helmholtz equation at low frequencies," IEEE Comput. Sci. Eng., vol. 5, pp. 32-38, Jul.-Sep. 1998.

[4] J.-S. Zhao and W. C. Chew, "Three dimensional multilevel fast multipole algorithm from static to electrodynamic," Microw. Opt. Technol. Lett., vol. 26, no. 1, pp. 43-48, Jul. 2000.

[5] J.-S. Zhao and W. C. Chew, "Applying LF-MLFMA to solve complex PEC structures," Microw. Opt. Technol. Lett., vol. 28, no. 3, pp. 155-160, Feb. 2001. 
[6] L. J. Jiang and W. C. Chew, "Low-frequency fast inhomogeneous planewave algorithm (LF-FIPWA)," Microw. Opt. Technol. Lett., vol. 40, no. 2, pp. 117-122, Jan. 2004.

[7] E. Darve and P. Have, "Efficient fast multipole method for low-frequency scattering," J. Comput. Phys., vol. 197, no. 1, pp. 341-363, Jan. 2004.

[8] Y.-H. Chu and W. C. Chew, "A multilevel fast multipole algorithm for electrically small composite structures," Microw. Opt. Technol. Lett., vol. 43, no. 3, pp. 202-207, Nov. 2004.

[9] I. Bogaert, D. Pissoort, and F. Olyslager, "A normalized plane wave method for 2D Helmholtz problems," Microw. Opt. Technol. Lett., vol. 48, no. 2, pp. 237-243, Feb. 2006.

[10] I. Bogaert, J. Peeters, and F. Olyslager, "A nondirective plane wave MLFMA stable at low frequencies," IEEE Trans. Antennas Propag. vol. 56, no. 12, pp. 3752-3767, Dec. 2008.

[11] I. Bogaert and F. Olyslager, "A low frequency stable plane wave addition theorem," J. Comput. Phys., vol. 228, no. 4, pp. 1000-1016, Mar. 2009.

[12] Ö. Ergül and L. Gürel, "Efficient solutions of metamaterial problems using a low-frequency multilevel fast multipole algorithm," Prog. Electromagn. Res., vol. 108, pp. 81-99, 2010.

[13] Ö. Ergül and B. Karaosmanoğlu, "Low-frequency fast multipole method based on multiple-precision arithmetic," IEEE Antennas Wireless Propag. Lett., vol. 13, pp. 975-978, 2014.

[14] Ö. Ergül and B. Karaosmanoğlu, "Approximate stable diagonalization of the Green's function for low frequencies," IEEE Antennas Wireless Propag. Lett., vol. 13, pp. 1054-1056, 2014.

[15] L. Xuan, A. Zhu, R. J. Adams, and S. D. Gedney, "A broadband multilevel fast multipole algorithm," in Proc. IEEE AP-S Int. Symp., 2004, vol. 2, pp. 1195-1198.

[16] E. Darve and P. Have, "A fast multipole method for Maxwell equations stable at all frequencies," Phil. Trans. R. Soc. Lond. A, vol. 362, pp. $603-$ 628, Mar. 2004

[17] H. Wallén and J. Sarvas, "Translation procedures for broadband MLFMA," Prog. Electromagn. Res., vol. 55, pp. 47-78, 2005.

[18] L. J. Jiang and W. C. Chew, "A mixed-form fast multipole algorithm," IEEE Trans. Antennas Propag., vol. 53, no. 12, pp. 4145-4156, Dec. 2005.

[19] H. Cheng, W. Y. Crutchfield, Z. Gimbutas, L. F. Greengard, J. F. Ethridge, J. Huang, V. Rokhlin, N. Yarvin, and J. Zhao, "A wideband fast multipole method for the Helmholtz equation in three dimensions," J. Comput. Phys., vol. 216, pp. 300-325, Jul. 2006.

[20] H. Wallén, S. Järvenpää, P. Ylä-Oijala, and J. Sarvas, "Broadband Müller-MLFMA for electromagnetic scattering by dielectric objects," IEEE Trans. Antennas Propag., vol. 55, no. 5, pp. 1423-1430, May 2007.

[21] M. Vikram, H. Huang, B. Shanker, and T. Van, "A novel wideband FMM for fast integral equation solution of multiscale problems in electromagnetics," IEEE Trans. Antennas Propag., vol. 57, no. 7, pp. 2094-2104, Jul. 2009.

[22] V. Melapudi, B. Shanker, S. Seal, and S. Aluru, "A scalable parallel wideband MLFMA for efficient electromagnetic simulations on large scale clusters," IEEE Trans. Antennas Propag., vol. 59, no. 7, pp. 2565 2577, Jul. 2011

[23] X.-M. Pan, J. Wei, Z. Peng, and X. Sheng, "A fast algorithm for multiscale electromagnetic problems using interpolative decomposition and multilevel fast multipole algorithm, Radio Sci., vol. 47, no. 1, pp. 111, Feb. 2012

[24] S. Järvenpää, J. Markkanen, and P. Ylä-Oijala, "Broadband multilevel fast multipole algorithm for electric-magnetic current volume integral equation," IEEE Trans. Antennas Propag., vol. 61, no. 8, pp. 4393 4397, Aug. 2013.

[25] J. G. Wei, Z. Peng, and J. F. Lee, "Multi-scale electromagnetic computations using a hierarchical multi-level fast multipole algorithm," Radio Sci., pp. 1-36, Jul. 2014

[26] W.-B. Kong, H.-X. Zhou, W.-D. Li, G. Hua, and W. Hong, "The MLFMA equipped with a hybrid tree structure for the multiscale EM scattering," Int. J. Antennas Propag., no. 281303, pp. 1-6, 2014.

[27] Ö. Ergül and B. Karaosmanoğlu, "Broadband multilevel fast multipole algorithm based on an approximate diagonalization of the Green's function," IEEE Trans. Antennas Propag., vol. 63, no. 7, pp. 3035-3041, Jul. 2015.

[28] M. Takrimi, Ö. Ergül, and V. B. Ertürk, "A novel broadband multilevel fast multipole algorithm with incomplete-leaf tree structures for multiscale electromagnetic problems," IEEE Trans. Antennas Propag. submitted for publication. 